\title{
ИНСТРУМЕНТАЛЬНАЯ ПАЛЬПАЦИЯ В ЭНДОСКОПИЧЕСКОЙ ХИРУРГИИ ПОЧЕК: ОПЫТ ПРИМЕНЕНИЯ
}

\author{
Р. Ф. Солодова ${ }^{1 凶}$, М. П. Толстых ${ }^{2}$, Т. К. Исаев ${ }^{3}$ Р. Н. Трушкин ${ }^{3}$, В. И. Вторенко ${ }^{3}$, В. М. Староверов ${ }^{1}$, М. Э. Соколов $^{1}$ \\ Механико-математический факультет, Московский государственный университет имени М. В. Ломоносова, Москва \\ 2 Московский государственный медико-стоматологический университет имени А. И. Евдокимова, Москва \\ ${ }^{3}$ Урологическое отделение, Городская клиническая больница № 52, Москва
}

\begin{abstract}
Пальпаторная оценка - один из классических методов исследования при открытых хирургических вмешательствах. В малоинвазивной хирургии интраоперационная мануальная пальпация невозможна при оценке тактильных характеристик тканей. В России единственным доступным прибором для интраоперационной оценки и объективной регистрации вязко-упругих характеристик тканей является медицинский тактильный эндохирургический комплекс (МТЭК). Целью работы было изучить возможности применения МТЭК в хирургии почек. Исследование проводили в ходе девяти плановых лапароскопических вмешательств: по поводу светлоклеточного рака почки и простых кист почки. Выявлены особенности, отличающие использование МТЭК в хирургии почек от его применения на органах гастроинтестинального тракта и легких. Ключевым фактором, определяющим наличие этих особенностей, является обратное соотношение вязко-упругих характеристик: исследованные опухоли оказались мягче окружающей ткани. Сделан вывод о невозможности выявления тактильными методами новообразований, расположенных в паренхиме. Для поверхностных новообразований в одном из девяти случаев механорецепторная пальпация позволила выявить четкое расположение границы опухоли. Применение МТЭК позволило количественно оценить и зафиксировать разницу в жесткостных характеристиках опухоли и неизмененной ткани, что открывает возможность исследования прогностической значимости объективно регистрируемых тактильных характеристик новообразований почки на основании полученных цифровых данных.
\end{abstract}

Ключевые слова: хирургия почек, инструментальная механорецепторная пальпация, объективная регистрация тактильного образа, медицинский тактильный эндохирургический комплекс (МТЭК), светлоклеточный рак почки, киста почки

Финансирование: работа выполнена при поддержке Российского научного фонда: проект № 16-11-00058 «Разработка методов и алгоритмов автоматизированного анализа медицинской тактильной информации и классификации тактильных образов».

Благодарности: авторы выражают благодарность А. В. Галатенко и В. В. Галатенко (МГУ имени М. В. Ломоносова) за замечания, ценные комментарии и помощь в подготовке текста.

$\bowtie$ Для корреспонденции: Розалия Фаилевна Солодова

Ленинские горы, д. 1, к. 46, г. Москва, 119991; rozaliya@solodov.org

Статья получена: 26.07.2018 Статья принята к печати: 23.11.2018

DOI: $10.24075 /$ vrgmu.2018.069

\section{INSTRUMENTAL PALPATION IN ENDOSCOPIC RENAL SURGERY: CASE REPORTS AND ANALYSIS}

\author{
Solodova RF ${ }^{1}$, Tolstykh $\mathrm{MP}^{2}$, Isaev $\mathrm{TK}^{3}$, Trushkin $\mathrm{RN}^{3}$, Vtorenko VI ${ }^{3}$, Staroverov VM${ }^{1}$, Sokolov ME ${ }^{1}$ \\ ${ }^{1}$ Faculty of Mechanics and Mathematics, Lomonosov Moscow State University, Moscow \\ ${ }^{2}$ Yevdokimov Moscow State University of Medicine and Dentistry, Moscow \\ ${ }^{3}$ City Clinical Hospital №52, Moscow Health Department, Moscow
}

Palpation is one of the classic examination methods in open surgeries. In minimally invasive surgery, intra-operational manual palpation is impossible to use for assessing tactile characteristics of tissues. In Russia, the only available instrument for intraoperational assessment and objective registration of tissue visco-elastic properties is the Medical Tactile Endosurgical Complex (MTEC). The aim of this work was to study the performance of MTEC in renal surgery. The study was performed during nine elective laparoscopic surgeries for clear cell renal carcinoma and simple renal cysts. We have found several differences in the use of MTEC in renal surgery, as compared to its use in gastrointestinal or lung surgeries. The key factor determining these differences was the inverse relations between tissue visco-elastic properties: the studied tumors were softer than the surrounding tissue. Detection of intraparenchymal tumors by tactile methods was impossible. For surface tumors, in one case out of nine it was possible to strictly locate the border of the tumor by tactile examination. We were able to quantitatively assess and determine the difference in hardness of tumors and intact tissue using MTEC. This allows studying the prognostic value of objectively registered tactile characteristics of renal tumors.

Keywords: renal surgery, instrumental mechanoreceptoric palpation, objective registration of tactile images, Medical Tactile Endosurgical Complex (MTEC), clear cell renal carcinoma, renal cyst

Funding: the work was done with the financial support of the Russian Science Foundation (project No. 16-11-00058 "Development of methods and algorithms for automated medical tactile information analysis and tactile image classification").

Acknowledgments: the authors thank Galatenko A.V. and Galatenko V.V. (Lomonosov Moscow State University) for valuable comments, criticism, and help with preparing the manuscript.

$\triangle$ Correspondence should be addressed: Rozaliya F. Solodova

Leninskie gory 1, bl. 46, Moscow, 119991; rozaliya@solodov.org

Received: 26.07.2018 Accepted: 23.11.2018

DOI: 10.24075/brsmu.2018.069 
Пальпаторное исследование, основанное на чувстве осязания, является одним из базовых этапов осмотра и рутинно применяется во время открытых хирургических вмешательств. Мануальная пальпация позволяет оценивать вязко-упругие свойства органов и тканей. Исследование основано на оценке тактильных характеристик, которые изменяются при различных патологических процессах. В частности, при развитии онкологических новообразований в большинстве случаев наблюдается увеличение жесткости [1].

C развитием малоинвазивных вмешательств интраоперационная оценка тактильных характеристик тканей изменилась: в эндоскопической хирургии она опосредована инструментарием и приобрела форму кинестетического ответа [2], а в робот-ассистированной хирургии фактически сведена на нет [3, 4]. Информативность обратной связи при манипуляциях существенно зависит от опыта и квалификации хирурга. Следует отметить, что даже традиционная пальпация, несмотря на ее длительное применение в медицинской практике, не является детально стандартизованной процедурой, и интерпретация ее результатов значимо зависит от опыта и тренированности врача $[5,6]$.

Разработка инструментальных методов пальпации позволит увеличить инсормированность оператора о тактильных свойствах тканей при малоинвазивных операциях и, в частности, позволит осуществлять такую оценку при робот-ассистированных операциях [7]. Кроме того, она потенциально позволяет объективизировать методику тактильного исследования, в том числе за счет возможности записи и воспроизведения полученной информации

В медицинской практике используют приборы для объективной оценки тактильных характеристик тканей и выявления новообразований методом тактильной механорецепторной диагностики в молочной и предстательной железах [8, 9]. Описано использование инструментального тактильного вагинального исследования для диагностики опущения тазовых органов [10] и инструментальной пальпации для выявления камней почек при их лапароскопической экстракции [11].

В России единственным коммерчески доступным прибором для интраоперационной оценки тактильных характеристик тканей является медицинский тактильный эндохирургический комплекс МТЭК-01 (АО НПО «СПЛАВ»; Россия). МТЭК используется, в частности, для выявления и локализации онкологических образований при периферических раках легкого и гастроинтестинальных злокачественных опухолях $[12,13]$.

Активное внедрение малоинвазивных методов лечения в хирургию почек приводит к росту необходимости применения инструментального тактильного исследования для точной локализации зоны патологических изменений и дополнительной оценки распространенности патологического процесса во время хирургических вмешательств. Целью настоящей работы было изучение возможности применения МТЭК-01 в хирургии почек.

\section{ПАЦИЕНТЫ И МЕТОДЫ}

\section{Пациенты}

В период с марта по май 2017 г. выполнено 9 хирургических вмешательств с использованием МТЭК-01 в ГБУЗ «Городская клиническая больница № 52 Департамента здравоохранения города Москвы». Исследование было одобрено этическим комитетом больницы (протокол № 0101/0117 от 25 января 2017 г.). Критерии включения в исследование: показания к плановому потенциально органосохраняющему лапароскопическому оперативному вмешательству (фенестрация кист, резекция почки) или диагностический этап операции лапароскопическим доступом на паренхиме почки (этап несрэктомии). Критерии исключения: отсутствие возможности и общих показаний к выполнению вмешательства лапароскопическим доступом. В исследовании приняли участие 9 пациентов (4 мужчин, 5 женщин) в возрасте 48-78 лет (средний возраст составил 63,7 года). После объяснения пациентам возможности применения дополнительных интраоперационных диагностических методов во время операции все они подписали информированное добровольное согласие на хирургическое вмешательство.

Хирургические вмешательства включали 7 резекций почек, одну нефрэктомию и одну плановую нефрадреналэктомию (см. таблицу).

Таблица. Клиническая характеристика пациентов, вид хирургического вмешательства и результаты гистологического исследования

\begin{tabular}{|c|c|c|c|c|c|c|}
\hline Пациент & Пол & Возраст & Диагноз & Лапароскопическая операция & $\begin{array}{c}\text { Гистологическое } \\
\text { описание }\end{array}$ & Размер образования (мм) \\
\hline 1 & M & 52 & $\begin{array}{l}\text { Рак левой почки T3aNOMO. } \\
\text { ХПН } 1 \text { ст. ХБП } 3 \text { ст. }\end{array}$ & Нефрэктомия слева & $\begin{array}{l}\text { Светлоклеточный рак } \\
\text { почки G2 по Fuhrman }\end{array}$ & 68 \\
\hline 2 & M & 66 & Рак левой почки T1bNOM0 & Нефрадреналэктомия слева & $\begin{array}{l}\text { Светлоклеточный рак } \\
\text { почки G3 по Fuhrman }\end{array}$ & 54 \\
\hline 3 & Ж & 78 & $\begin{array}{l}\text { Рак единственной правой } \\
\text { почки T1aN0M0 }\end{array}$ & $\begin{array}{l}\text { Резекция единственной правой } \\
\text { почки с опухолью }\end{array}$ & $\begin{array}{l}\text { Светлоклеточный рак } \\
\text { почки G1 по Fuhrman }\end{array}$ & 34 \\
\hline 4 & Ж & 77 & $\begin{array}{l}\text { Киста верхнего сегмента } \\
\text { правой почки Bosniak IIF }\end{array}$ & $\begin{array}{l}\text { Резекция верхнего полюса } \\
\text { правой почки со стенкой кисты }\end{array}$ & $\begin{array}{l}\text { Простая напряженная } \\
\text { киста почки }\end{array}$ & 62 \\
\hline 5 & Ж & 48 & $\begin{array}{l}\text { Киста верхнего сегмента } \\
\text { правой почки Bosniak III }\end{array}$ & $\begin{array}{l}\text { Резекция правой почки } \\
\text { со стенкой кисты }\end{array}$ & Простая киста почки & 86 \\
\hline 6 & Ж & 53 & $\begin{array}{l}\text { Киста нижнего сегмента } \\
\text { левой почки Bosniak IIF }\end{array}$ & $\begin{array}{l}\text { Резекция левой почки } \\
\text { со стенкой кисты }\end{array}$ & Простая киста почки & 79 \\
\hline 7 & M & 63 & $\begin{array}{l}\text { Киста нижнего полюса } \\
\text { левой почки Bosniak IIF }\end{array}$ & $\begin{array}{l}\text { Резекция левой почки } \\
\text { со стенкой кисты }\end{array}$ & Простая киста почки & 64 \\
\hline 8 & M & 68 & $\begin{array}{l}\text { Киста среднего сегмента } \\
\text { левой почки Bosniak III }\end{array}$ & $\begin{array}{l}\text { Резекция левой почки } \\
\text { со стенкой кисты }\end{array}$ & Простая киста почки & 57 \\
\hline 9 & Ж & 68 & $\begin{array}{l}\text { Киста верхнего сегмента } \\
\text { левой почки Bosniak IIF }\end{array}$ & $\begin{array}{l}\text { Резекция левой почки } \\
\text { со стенкой кисты }\end{array}$ & Простая киста почки & 41 \\
\hline
\end{tabular}




\section{Описание МТЭК}

Для проведения инструментального тактильного исследования во время хирургических вмешательств использовали МТЭК-01 (АО НПО «СПЛАВ»; Россия), состоящий из тактильных механорецепторов (зондов), компьютера со специально разработанным программным обеспечением и опционального тактильного дисплея, с которого хирург может считывать тактильные образы рукой. Прибор производится с двумя вариантами диаметра рабочей части тактильного зонда - 20 и 10 мм. На рабочей поверхности зондов (в зависимости от диаметра) находятся 19 или 7 датчиков давления, беспроводным образом до 100 раз в секунду передающих данные на компьютер. На рис. 1 представлен зонд диаметром 10 мм с 7 датчиками на рабочей поверхности. Кроме воспроизведения в режиме реального времени на тактильном дисплее результать исследования отображаются визуально с использованием цветовой адаптивной шкалы. При средней силе нажатия мягкие ткани отображаются зеленым цветом, твердые красным, а синий соответствует промежуточным результатам.

\section{Обучение хирургической бригады}

На этапе освоения и внедрения нового типа прибора в клиническую практику необходимо проведение обучения как в теоретическом, так и в практическом ключе. Для обеспечения воспроизводимости результатов эффективно проведение обучения на тренажере: все члены хирургической бригады (не только оперирующий хирург) нарабатывали навыки инструментальной пальпации на коробочном тренажере. Цели обучения включали формирование объективного представления о возможностях метода без завышенных ожиданий, получение навыков трактовки адаптивной цветовой шкалы и оценки количества допускаемых ошибок при распознании твердого объекта в мягких тканях. В обучении принимали участие опытные практикующие врачи-хирурги. Продолжительность обучения составляла не более одного академического часа. В последующем все исследования выполнял один хирург, при необходимости ассистенты тоже могли применить методику. Подготовка состояла из брифинга с объяснением материальнотехнической базы, инструментальной пальпации объектов разной твердости (не скрытых в массе ткани) и пальпации металлического шарика, погруженного в мягкую губочную массу. Все хирурги освоили методику за 5-минутное обучение за единственным исключением: одному хирургу потребовался дополнительный индивидуальный тренинг. С учетом этого случая шести подходов к лапароскопическому коробочному тренажеру оказалось достаточно для достижения поставленных в рамках обучения целей.

Результаты обучения подтвердили, что информация, получаемая хирургом при инструментальной тактильной пальпации, не является полным аналогом информации, получаемой при традиционной пальпации. В процессе использования МТЭК хирург объединяет кинестетические ощущения от контакта с тканями, визуальную картину пальпируемого органа, визуализацию тактильного образа на основе адаптивной цветовой шкалы и, опционально, воспроизведение тактильного образа на тактильном дисплее. Хирург анализирует эту совокупность информации для получения ответов на вопросы, определяемые целью проводимого исследования (например, поиск края опухоли для обеспечения необходимого и достаточного объема резекции).

\section{Ход исследования}

МТЭК применен у пациентов, которым по медицинским урологическим показаниям планировали лапароскопическую резекцию почки, фенестрацию кист либо нефрэктомию. Тактильные исследования выполнял один хирург в составе постоянной лапароскопической хирургической бригады. План операции составляли с учетом того, что продление времени вмешательства и наркоза возможно не более чем на 10-15 мин. Зону пальпации всегда визуально контролировали. Паранефрий над зоной исследования снимали, что соответствовало стандартному протоколу операции. Влияние инструментальной механорецепторной пальпации на изменение изначально намеченного плана операции отсутствовало. Для интраоперационной верификации расположения патологического образования и контроля результатов инструментального тактильного исследования проводили интраоперационное исследование ультразвуковым прибором Flex Focus (BK Medical; Дания). Этапы вмешательства, связанные с временным прекращением кровотока в почке (при резекции), не потребовали увеличения времени. Соответственно хирург во время инструментального тактильного исследования не подвергался временному стрессу, обусловленному использованием новой технологии. Дополнительные порты, помимо установленных для исполнения стандартного протокола лапароскопического вмешательства, не применяли.

Условия инструментальной пальпации различались в зависимости от характеристик образования. Исследование визуально различимых образований проводили от условной точки, ближайшей к воротам почки, по часовой стрелке до полной локализации образования. Использовали два способа оценки: статическую и динамическую. При статической оценке тактильный зонд пошагово прикладывали к разным участкам исследуемой ткани. При динамическом исследовании зонд перемещали под небольшим давлением по ткани, при этом контакт датчиков с тканью постоянно сохраняли. При визуально неразличимых границах инструментальное тактильное исследование начинали от верхнего полюса предполагаемой зоны и продолжали по часовой стрелке. В ходе исследования: 1) анализировали возможность выявить границу образования на основе тактильной механорецепторной информации; 2) давали тактильную характеристику опухоли; 3) фиксировали кинестетическое

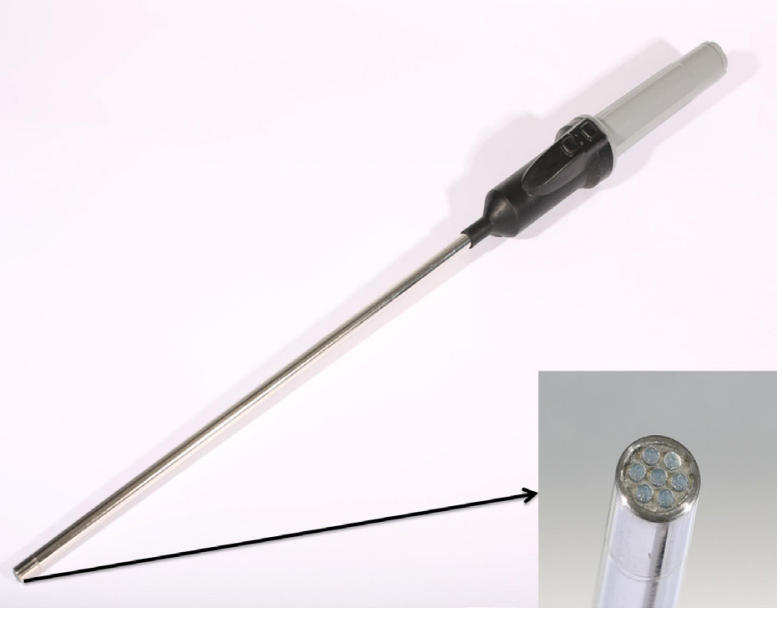

Рис. 1. Тактильный зонд диаметром 10 мм с 7 датчиками давления на рабочей поверхности 
ощущение (мягкая-твердая, мягче-тверже здоровой почечной ткани)

При визуализации тактильного образа на основе адаптивной цветовой шкалы выделяли следующие шаблоны:

- дряблый: центр выведен из пальпации за счет давления на внешний периметр рабочей поверхности механорецептора (центр зеленее) (рис. 2А);

- упругий: центр под давлением, внешний периметр частично выведен из пальпации (центр синий или красный, периметр соответствует меньшему давлению) (рис. 2Б);

- край: имеется разделительная полоса без острых углов, с каждой стороны от которой регистрируемые значения давления близки к однородным (но давление по разные стороны от полосы визуализируется различными оттенками) (рис. 2В).

Учитывая стандартное расположение лапароскопических портов, оптимального угла контакта (когда тактильный зонд практически перпендикулярен поверхности изучаемой ткани) достигали при исследовании преимущественно передней, медиальной и частично прочих боковых поверхностей почки. Инструментальная пальпация задней поверхности почки требовала мобилизации почки и вращения ее ножки. С учетом этого обстоятельства во избежание ишемического повреждения инструментальную пальпацию задней поверхности выполняли только в случаях, когда планировали удаление органа.

\section{РЕЗУЛЬТАТЫ ИССЛЕДОВАНИЯ}

При исследовании основное внимание уделяли:

- возможности «пальпаторной визуализации» новообразования в почке для образований, расположенных поверхностно или в глубине паренхимы;

- возможности влияния вязко-упругих свойств окружающего субстрата (характеристика паренхимы) на информативность инструментального тактильного исследования;

- сопоставимости результатов инструментального тактильного исследования с информацией, получаемой посредством кинестетической обратной связи.

Были получены следующие результаты.

Пациент По., 52 года. Макроскопически солидная бугристая опухоль размером до 68 мм расположена поверхностно и визуально определяется при лапароскопии (рис. 3). Кинестетически твердая. По кинестетическим ощущениям опухоль почки мягче окружающей неизмененной ткани, что согласовывалось с визуализацией регистрируемого тактильного образа на основе адаптивной цветовой шкалы (рис. 3В). Граница инструментальным тактильным методом определялась убедительно. Следует отметить, что в силу размеров опухоли угол исследования тактильным зондом на опухоли значительно отличался от угла в неизмененной почке.

Пациент Пе., 66 лет. Макроскопически кистозная опухоль размером 54 мм расположена поверхностно и визуально определяется при лапароскопии. Кинестетически мягкая. По кинестетическим ощущениям паренхима почки плотнее опухоли, по инструментально регистрируемым тактильным характеристикам опухоль также мягче паренхимы. Четкое определение границы патологии на основе инструментального тактильного исследования признано невозможным.

Пациент С., 78 лет. Опухоль в верхнем полюсе почки размером 34 мм (T1aNOMO). Выполнена органосохраняющая резекция единственной почки. При подкапсульном расположении опухоли она не была различима визуально и не локализовывалась инструментальным тактильным исследованием (рис. 4). Резекция стала технически исполнимой только после ультразвуковой визуализации опухоли с 3D-реконструкцией, подтвердившей резектабельность и позволившей определить объем необходимого оперативного приема. При этом было обнаружено, что глубина залегания образования всего 2 мм (рис. 4А). Длительность хирургического вмешательства составила 140 мин, временный контроль гемостаза осуществлен наложением эндоскопически клипсы «бульдог» на всю ножку, этап тепловой ишемии составил 17 мин. Окончательный гемостаз достигнут прошиванием раны почки.

Пациент Ш., 77 лет. Макроскопически поверхностно расположенная напряженная киста размером 62 мм, хорошо определяемая визуально. Кинестетически мягкая, по кинестетическим ощущениям мягче паренхимы почки, по тактильным характеристикам в адаптивной цветовой шкале практически не отличается от здоровой ткани и выводится с трудом: при умеренном давлении все соты в зеленом диапазоне.

Оставшиеся пять случаев макро- и микроскопически были представлены простыми ненапряженными кистами, поэтому объединены в одну группу. Во всех пяти случаях четкое определение границы кист на основе информации, получаемой при инструментальном механорецепторном тактильном исследовании, было признано невозможным.

Кистам соответствовал шаблон с однородно окрашенным периметром, по которому на основе соотнесения оттенков центральной соты и периферии оказывался возможным анализ напряженности: напряженной кисте соответствовал упругий шаблон, ненапряженным - промежуточные между выраженным дряблым и выраженным упругим.

\section{ОБСУЖДЕНИЕ РЕЗУЛЬТАТОВ}

После описания первой лапароскопической несрэктомии [14] началась эра малоинвазивных вмешательств в хирургии почек. Преимущества эндоскопии, связанные с меньшей травматизацией, сокращением послеоперационного периода,
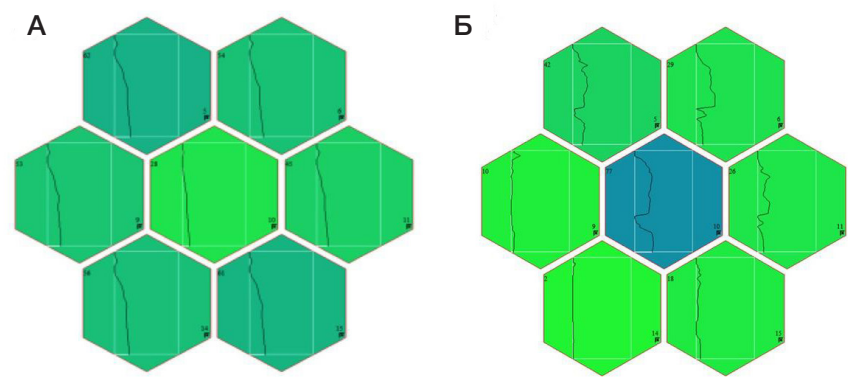

B

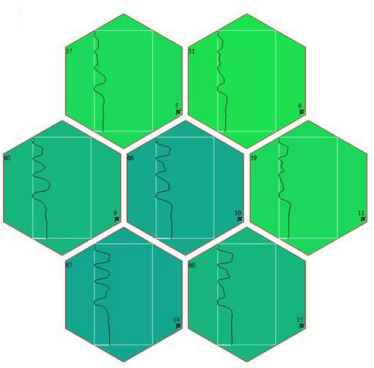

Рис. 2. Визуализация характерных тактильных кадров в адаптивной цветовой шкале: шаблон дряблый (А); шаблон упругий (Б); шаблон края образования (B) 
улучшением визуализации во время операции за счет технических средств, сопрягаются с сокращением спектра методов исследования в силу потери возможности пропальпировать органы и ткани. В эндоскопической хирургии единственной обратной связью является кинестетическая, определяемая силой воздействия на мышцы и связки [2]. В стандартной робот-ассистированной хирургии отсутствует и тактильная, и кинестетическая обратная связь [3]. Исследования с применением дополнительных устройств для создания ответа тканей показали, что при их использовании сокращается сила захвата и, как следствие, уменьшается повреждение тканей [15].

В литературе описаны различные виды приборов, разработанных для получения кинестетической и тактильной информации во время лапароскопических вмешательств, включая робот-ассистированные операции [12, 13, 16-19]. Значительная их часть позволяет решать лишь узкие задачи, например контролировать силу захвата, и не обеспечивает возможности инструментального тактильного исследования. Напротив, МТЭК предназначен именно для проведения механорецепторной пальпации.

Помимо возможности получения дополнительной информации при эндоскопических вмешательствах инструментальная пальпация потенциально позволяет объективизировать результаты тактильного исследования, что в свою очередь увеличивает эффективность исследования на начальных этапах накопления опыта специалистом.

Внедрение объективного метода оценки тактильных характеристик тканей во время хирургических вмешательств кажется перспективным, но малоизученным направлением в хирургии почек. В рамках исследования была изучена применимость МТЭК в хирургии почек для проведения инструментальной механорецепторной пальпации. Были выявлены особенности, отличающие использование МТЭК для инструментальной тактильной пальпации в хирургии почек от его применения на органах гастроинтестинального тракта и легких [12, 13]. Ключевым фактором, определяющим наличие этих особенностей, является обратное соотношение вязко-упругих характеристик онкологических новообразований в почке и окружающей ткани. В то время как большинство злокачественных образований оказывается более жесткими, чем неизмененная ткань [1, 20-22], исследованные опухоли почек (по результатам гистологического исследования все случаи были представлены светлоклеточным раком) оказались мягче окружающей неизмененной ткани. Эти результаты согласуются с наблюдениями, показавшими, что модуль Юнга здоровой ткани почки значимо превосходит модуль Юнга почечно-клеточной карциномы [23]. Принципиальность разницы прямого и обратного соотношений жесткости новообразования и окружающей ткани объясняется тем, что даже при проведении мануальной пальпации характеристика мягкости оценивается не в отдельности, а в совокупности получаемых ощущений. В силу этого образования, расположенные в паренхиме даже при небольшой глубине в 2 мм, из-за их мягкой консистенции оказываются невыявляемыми с помощью тактильных методов, так как более плотная ткань паренхимы почек скрывает мягкие включения. Уместно привести следующую «кулинарную» аналогию: при изготовлении выпечки готовность изделия не может быть оценена по тактильным характеристикам, так как схватившаяся корочка одинаково пружинит вне зависимости от степени испеченности внутренней части, и для проверки готовности используются нетактильные методы. В то же время поверхностно расположенные онкологические новообразования тактильным инструментальным исследованием идентифицировались: в одном из случаев тактильное исследование обеспечило четкую локализацию границы.

Кисты по инструментально регистрируемым тактильным характеристикам фрактически не отличались от нормальной ткани почки, что хорошо согласовывалось с опосредованной пальпацией инструментарием и в последующем с мануальной пальпацией удаленного материала. В случае напряженной кисты ее плотность была незначительно выше, чем у здоровой паренхимы,
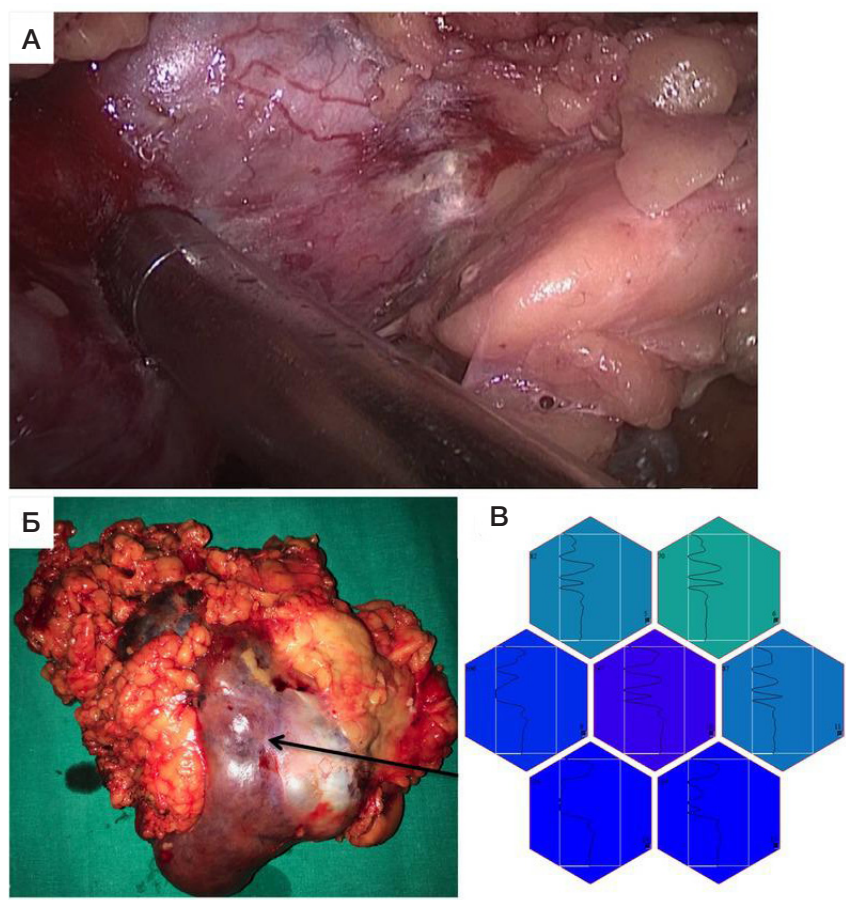

Рис. 3. Исследование опухоли с визуально различимой границей А. Изображение с лапароскопической камеры, исследование тактильным зондом. Б. Удаленный макропрепарат, обозначена визуальная граница опухоли. В. Визуализация тактильного кадра в адаптивной цветовой шкале
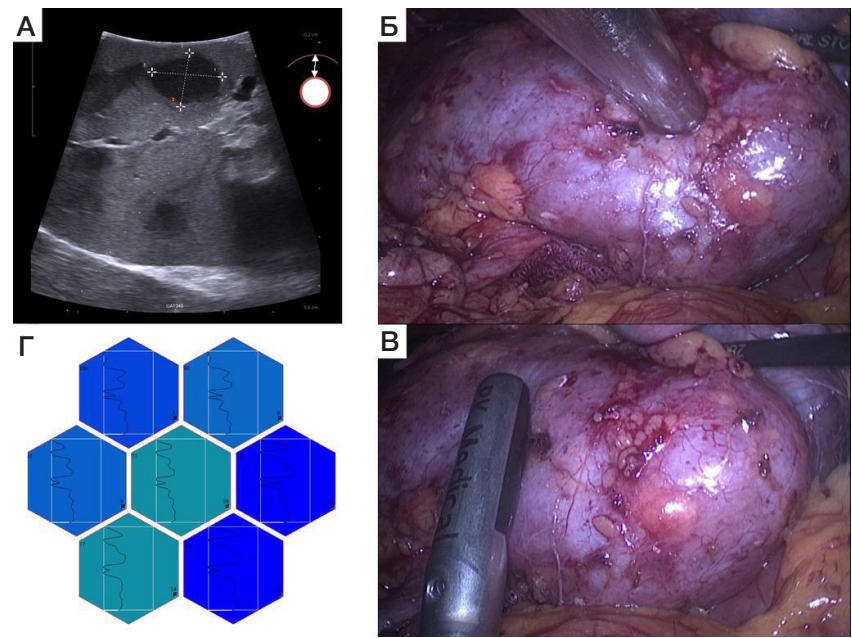

Рис. 4. Исследование визуально не определяемой опухоли в паренхиме. А. Ультразвуковое исследование опухоли с отображением расстояния от края. Б. Исследование тактильным зондом: изображение с лапароскопической камеры. В. Сканирование ультразвуковым датчиком: изображение с лапароскопической камеры. Г. Визуализация тактильного кадра в адаптивной цветовой шкале 
но тактильно локализовать границу патологического процесса не удалось.

Важным и полезным наблюдением мы считаем повышение информативности инструментального тактильного исследования с помощью МТЭК за счет применения динамической, а не статической пальпации. Ключевым отличием динамической пальпации является «перекатывание» рабочей поверхности инструмента от точки установки в исследуемой области. С учетом фиксации прибора в троакаре степень свободы для динамической пальпации была ограниченной, но достаточной для выполнения приема. Статическая пальпация с приложением давления по оси прибора оказывалась значительно менее информативной.

В процессе обучения на экспериментальной модели металлического шарика в губочной массе было выявлено, что локализация с помощью МТЭК упругих вкраплений, по размеру меньших рабочей поверхности зонда, проще, чем локализация вкраплений большого размера. Это обстоятельство позволяет прогнозировать увеличение эффективности применения МТЭК, если получится увеличить площадь рабочей поверхности его зонда без увеличения диаметра самого прибора.

Конкретно для почечной хирургии кажется необходимым внесение в программную составляющую МТЭК изменений, обеспечивающих возможность переключения на режим поиска новообразований, которые мягче окружающей ткани: текущее программное обеспечение ориентировано именно на поиск более твердых включений [24, 25]. В частности, простое изменение адаптивной цветовой шкалы, выделяющее при визуализации более мягкие зоны, уже увеличило бы естественность восприятия информации хирургом.

Несмотря на невозможность в большинстве случаев локализовать границы новообразований, опираясь на результаты исключительно инструментальной тактильной пальпации с использованием МТЭК, исследование показало различия в регистрируемых тактильных шаблонах при разных плотностях новообразований по сравнению с окружающей тканью. При стандартизации инструментальной регистрации тактильной информации (например, выделении тактильных кадров, соответствующих силе, в точности определяемой собственным весом тактильного зонда) это поднимает вопрос об изучении прогностической значимости инструментально регистрируемых тактильных характеристик для стадирования и уточнения тактики хирургического вмешательства. Для онкопатологий ряда органов такая связь уже известна $[22,26,27]$.

\section{ВЫВОДЫ}

Особенность онкологических новообразований почки, принципиальная для проведения инструментального тактильного исследования, заключается в обратном соотношении плотности новообразования и неизмененной ткани: новообразования являются менее плотными относительно ткани почек. Эта особенность приводит к невозможности выявления тактильными методами новообразований, расположенных в паренхиме, даже на небольшой глубине. Для поверхностных новообразований в одном случае информации, получаемой при механорецепторной пальпации, было достаточно для четкой локализации границы. В остальных случаях четкая локализация границы на основе инструментально регистрируемой тактильной информации была невозможна, однако применение МТЭК позволяло количественно оценить разницу плотности новообразования и неизмененной ткани. Появление технологии, позволяющей проводить такую оценку, открывает возможность исследования прогностической значимости объективно регистрируемых тактильных характеристик новообразований почки для интраоперационной экспресс-диагностики. В рамках исследования уточнена методология тактильного исследования, а также выработаны шаблоны для описания инструментальной тактильной диагностики почек.

\section{Литература}

1. Paszek MJ, Zahir N, Johnson KR, Lakins JN, Rozenberg Gl, Gefen A, et al. Tensional homeostasis and the malignant phenotype. Cancer Cell. 2005; 8 (3): 241-54.

2. Abdul-Muhsin HM, Humphreys MR. Advances in laparoscopic urologic surgery techniques. F1000Res. 2016; 5(F1000 Faculty Rev): 716.

3. Okamura AM. Haptic feedback in robot-assisted minimally invasive surgery. Curr Opin Urol. 2009; 19 (1): 102-7.

4. Lim SC, Lee HK, Park J. Role of combined tactile and kinesthetic feedback in minimally invasive surgery. Int J Med Robot. 2015; 11 (3): 360-374.

5. Fletcher SW, O'Malley MS, Pilgrim CA, Gonzalez JJ. How do women compare with internal medicine residents in breast lump detection? A study with silicone models. J Gen Intern Med. 1989; 4 (4): 277-83

6. McDonald S, Saslow D, Alciati MH. Performance and reporting of clinical breast examination: $A$ review of the literature. $C A$ : $A$ Cancer J Clin. 2004; 54 (6): 345-361.

7. Lim SC, Lee HK, Park J. Role of combined tactile and kinesthetic feedback in minimally invasive surgery. Int J Med Robot. 2015; 11 (3): 360-374

8. Egorov V, Sarvazyan AP. Mechanical imaging of the breast. IEEE Trans Med Imaging. 2008; 27 (9): 1275-87.

9. Weiss RE, Egorov V, Ayrapetyan S, Sarvazyan N, Sarvazyan A. Prostate mechanical imaging: a new method for prostate assessment. Urology. 2008; 71 (3): 425A.9.

10. Egorov V, van Raalte $\mathrm{H}$, Lucente V. Quantifying vaginal tissue elasticity under normal and prolapse conditions by tactile imaging Int Urogynecol J. 2012; 23 (4): 459-66.

11. Afshari E, Najarian S, Simforoosh N. Application of artificial tactile sensing approach in kidney-stone-removal laparoscopy. Biomed Mater Eng. 2010; 20 (5): 261-7.

12. Barmin V, Sadovnichy V, Sokolov M, Pikin O, Amiraliev A. An original device for intraoperative detection of small indeterminate nodules. Eur J Cardiothorac Surg. 2014; 46: 1027-1031.

13. Solodova RF, Galatenko W, Nakashidze ER, Shapovalyants SG, Andreytsev IL, Sokolov ME. et al. Instrumental mechanoreceptoric palpation in gastrointestinal surgery. Minimally invasive surgery. 2017; 2017.

14. Clayman RV, Kavoussi LR, Soper NJ, Dierks SM, Merety K,S, Darcy MD, et al. Laparoscopic nephrectomy: initial case report. J Urol. 1991; 146 (2): 278-282.

15. Wottawa CR, Genovese B, Nowroozi BN, Hart SD, Bisley JW, Grundfest WS, et al. Evaluating tactile feedback in robotic surgery for potential clinical application using an animal model. Surg Endosc. 2016; 30 (8): 3198-209.

16. Puangmali P, Althoefer K, Seneviratne LD, Murphy D, Dasgupta P. State-of-the-art in force and tactile sensing for minimally invasive surgery. IEEE Sensors J. 2008; 8 (4): 371-381.

17. Dahiya RS, Metta G, Valle M, Sandini G. Tactile sensing — From humans to humanoids. IEEE Trans Robot. 2010; 26 (1): 1-20.

18. Schostek S, Schurr MO, Buess GF. Review on aspects of artificial 
tactile feedback in laparoscopic surgery. Med Eng Phys. 2009; 31 (8): 887-898.

19. Schostek S, Zimmermann M, Schurr MO, Prosst RL. Design and Performance of a Low-Cost Telemetric Laparoscopic Tactile Grasper. Surg Innov. 2016; 23 (3): 291-7.

20. Samani A, Zubovits J, Plewes D. Elastic moduli of normal and pathological human breast tissues: an inversion-techniquebased investigation of 169 samples. Phys Med Biol. 2007; 52 (6): 1565-76.

21. Hoyt K, Castaneda B, Zhang M, Nigwekar P, di Sant'agnese PA, Joseph JV, et al. Tissue elasticity properties as biomarkers for prostate cancer. Cancer Biomark. 2008; 4 (4-5): 213-25.

22. Kawano S, Kojima M, Higuchi Y, Sugimoto M, Ikeda K, Sakuyama N, et al. Assessment of elasticity of colorectal cancer tissue, clinical utility, pathological and phenotypical relevance. Cancer Sci. 2015; 106 (9): 1232-9.

23. Lee JW, Lorenzo El, Ahn B, Oh CK, Kim HJ, Han WK, et al. Palpation device for the identification of kidney and bladder cancer: a pilot study. Yonsei Med J. 2011; 52 (5): 768-72.
24. Solodova R, Staroverov V, Galatenko V, Galatenko A, Solodov E, Antonov A., et al. Automated detection of heterogeneity in medical tactile images. In: Westwood JD, Westwood SW, Fellander-Tsai L, Fidopiastis CM, Liu A, Senger S, et al, editors. Medicine Meets Virtual Reality 22. Vol. 220 of Studies in Health Technology and Informatics. Amsterdam: IOS Press. 2016; 383-389.

25. Solodova RF, Galatenko W, Nakashidze ER, Andreytsev IL, Galatenko AV, Senchik DK, et al. Instrumental tactile diagnostics in robot-assisted surgery. Med Devices (Auckl). 2016; 9: 377-382.

26. Evans A, Armstrong S, Whelehan P, Thomson K, Rauchhaus P, Purdie C, et al. Can shear-wave elastography predict response to neoadjuvant chemotherapy in women with invasive breast cancer? Br J Cancer. 2013; 109 (11): 2798-802.

27. Fenner J, Stacer AC, Winterroth F, Johnson TD, Luker KE, Luker GD. Macroscopic stiffness of breast tumors predicts metastasis. Sci Rep. 2014; 4: 5512.

\section{References}

1. Paszek MJ, Zahir N, Johnson KR, Lakins JN, Rozenberg GI, Gefen A, et al. Tensional homeostasis and the malignant phenotype. Cancer Cell. 2005; 8 (3): 241-54.

2. Abdul-Muhsin HM, Humphreys MR. Advances in laparoscopic urologic surgery techniques. F1000Res. 2016; 5(F1000 Faculty Rev): 716.

3. Okamura AM. Haptic feedback in robot-assisted minimally invasive surgery. Curr Opin Urol. 2009; 19 (1): 102-7.

4. Lim SC, Lee HK, Park J. Role of combined tactile and kinesthetic feedback in minimally invasive surgery. Int J Med Robot. 2015; 11 (3): 360-374

5. Fletcher SW, O'Malley MS, Pilgrim CA, Gonzalez JJ. How do women compare with internal medicine residents in breast lump detection? A study with silicone models. J Gen Intern Med. 1989; 4 (4): 277-83

6. McDonald S, Saslow D, Alciati MH. Performance and reporting of clinical breast examination: A review of the literature. CA: A Cancer J Clin. 2004; 54 (6): 345-361.

7. Lim SC, Lee HK, Park J. Role of combined tactile and kinesthetic feedback in minimally invasive surgery. Int J Med Robot. 2015; 11 (3): 360-374.

8. Egorov V, Sarvazyan AP. Mechanical imaging of the breast. IEEE Trans Med Imaging. 2008; 27 (9): 1275-87.

9. Weiss RE, Egorov V, Ayrapetyan S, Sarvazyan N, Sarvazyan A. Prostate mechanical imaging: a new method for prostate assessment. Urology. 2008; 71 (3): 425A.9.

10. Egorov V, van Raalte $\mathrm{H}$, Lucente V. Quantifying vaginal tissue elasticity under normal and prolapse conditions by tactile imaging. Int Urogynecol J. 2012; 23 (4): 459-66.

11. Afshari E, Najarian S, Simforoosh N. Application of artificial tactile sensing approach in kidney-stone-removal laparoscopy. Biomed Mater Eng. 2010; 20 (5): 261-7.

12. Barmin V, Sadovnichy V, Sokolov M, Pikin O, Amiraliev A. An original device for intraoperative detection of small indeterminate nodules. Eur J Cardiothorac Surg. 2014; 46: 1027-1031.

13. Solodova RF, Galatenko W, Nakashidze ER, Shapovalyants SG, Andreytsev IL, Sokolov ME. et al. Instrumental mechanoreceptoric palpation in gastrointestinal surgery. Minimally invasive surgery. 2017; 2017.

14. Clayman RV, Kavoussi LR, Soper NJ, Dierks SM, Merety K,S, Darcy MD, et al. Laparoscopic nephrectomy: initial case report. $J$ Urol. 1991; 146 (2): 278-282.

15. Wottawa CR, Genovese B, Nowroozi BN, Hart SD, Bisley JW, Grundfest WS, et al. Evaluating tactile feedback in robotic surgery for potential clinical application using an animal model. Surg Endosc. 2016; 30 (8): 3198-209.

16. Puangmali P, Althoefer K, Seneviratne LD, Murphy D, Dasgupta P. State-of-the-art in force and tactile sensing for minimally invasive surgery. IEEE Sensors J. 2008; 8 (4): 371-381.

17. Dahiya RS, Metta G, Valle M, Sandini G. Tactile sensing - From humans to humanoids. IEEE Trans Robot. 2010; 26 (1): 1-20.

18. Schostek S, Schurr MO, Buess GF. Review on aspects of artificial tactile feedback in laparoscopic surgery. Med Eng Phys. 2009; 31 (8): 887-898.

19. Schostek S, Zimmermann M, Schurr MO, Prosst RL. Design and Performance of a Low-Cost Telemetric Laparoscopic Tactile Grasper. Surg Innov. 2016; 23 (3): 291-7.

20. Samani A, Zubovits J, Plewes D. Elastic moduli of normal and pathological human breast tissues: an inversion-techniquebased investigation of 169 samples. Phys Med Biol. 2007; 52 (6): 1565-76.

21. Hoyt K, Castaneda B, Zhang M, Nigwekar P, di Sant'agnese PA, Joseph JV, et al. Tissue elasticity properties as biomarkers for prostate cancer. Cancer Biomark. 2008; 4 (4-5): 213-25.

22. Kawano S, Kojima M, Higuchi Y, Sugimoto M, Ikeda K, Sakuyama N, et al. Assessment of elasticity of colorectal cancer tissue, clinical utility, pathological and phenotypical relevance. Cancer Sci. 2015; 106 (9): 1232-9.

23. Lee JW, Lorenzo El, Ahn B, Oh CK, Kim HJ, Han WK, et al. Palpation device for the identification of kidney and bladder cancer: a pilot study. Yonsei Med J. 2011; 52 (5): 768-72.

24. Solodova R, Staroverov V, Galatenko V, Galatenko A, Solodov E, Antonov A., et al. Automated detection of heterogeneity in medical tactile images. In: Westwood JD, Westwood SW, Fellander-Tsai L, Fidopiastis CM, Liu A, Senger S, et al, editors. Medicine Meets Virtual Reality 22. Vol. 220 of Studies in Health Technology and Informatics. Amsterdam: IOS Press, 2016; 383-389.

25. Solodova RF, Galatenko W, Nakashidze ER, Andreytsev IL, Galatenko AV, Senchik DK, et al. Instrumental tactile diagnostics in robot-assisted surgery. Med Devices (Auckl). 2016; 9: 377-382.

26. Evans A, Armstrong S, Whelehan P, Thomson K, Rauchhaus $P$, Purdie C, et al. Can shear-wave elastography predict response to neoadjuvant chemotherapy in women with invasive breast cancer? Br J Cancer. 2013; 109 (11): 2798-802.

27. Fenner J, Stacer AC, Winterroth F, Johnson TD, Luker KE, Luker GD. Macroscopic stiffness of breast tumors predicts metastasis. Sci Rep. 2014; 4: 5512. 